\title{
Flexible Protocol Stacks
}

\author{
Christian Tschudin, University of Geneva \\ Centre Universitaire d'Informatique: 12, rue du Lac \\ <tschudin@cui.unige.ch>
}

Current standard protocol 'stack' architectures are static. The fixed number - for instance - of layers an application can access, or the order in which they must be arranged, exclude a large class of potential communication protocols. We propose to investigate more flexible protocol stacks consisting of a 'protocol environment' populated by standard communication functionalities. The main ingredient is the ability for an application to compose protocol stacks out of standard protocol entities as well as the downline loading of new protocol entities. This leads to application-tailored, extensible stacks, possibly established at run-time by a bootstrap procedure. Different ways to implement such an environment are discussed, and the current work on a 'toy environment' is presented.

Key words: computer communication, reference model, flexible protocol stacks, ComScript, communication bootstrap, PascalCom.

\section{Introduction}

The tremendous progress in the development of telecommunication applications is to a large degree due to the systematic use of layering. However, recent research also made appear some problems related to the present state of standardisation of layered protocol stacks. The implementation of the Remote Procedure Call concept (RPC) provides an example for this. In the OSI protocol suite [1] as well as with TCP/IP [2,3], RPC is treated as a high-level service because of its multiple needs for support by other services

Permission to copy without fee all or part of this material is granted provided that the copies are not made or distributed for direct commercial advantage, the ACM copyright notice and the title of the publication and its date appear, and notice is given that copying is by permission of the Association for Computing Machinery. To copy otherwise, or to republish, requires a fee and/or specific permission.

- 1991 ACM 0-89791-444-9/91/0008/0197...\$1.50 (e.g. data integrity, addressing and presentation). Therefore OSI and TCP/IP position RPC on the very top of their protocol stack. Amoeba (a distributed operating system [4]), on the other hand, considers RPC as the basic communication mechanism: a single optimized and monolithic protocol incorporates all required services (like data integrity etc.), thus violating among others the separation of these functions as it is defined in "open systems" concepts. While there is no problem to run OSI or TCP/IP protocols and applications in such operating systems, it is clear that applications relying on these protocol suites will not be able to directly take advantage of the communication mechanisms offered by the underlying system because these services can not be made 'visible' within the standard protocol suites.

In a first Section we will examine certain properties of layers within 'static' protocol stack architectures - some familiarity with OSI and TCP/IP concepts is required. The second Section introduces a more flexible model trying to overcome the limitations discussed in Section one. Finally we present PascalCom, a language which is based on Pascal and which allows to explore techniques needed for the handling of flexible protocol stacks.

\section{Description of the problem}

The concept of layering functionalities is a well-established paradigm: In the field of protocol standardisation it is probably the most elaborated and dominant structuring force. Each layer can be characterised by the set of services that become available at the layer's upper border. Layers are stacked, so that a layer can provide its new or enhanced services by building on services furnished by the layer underneath. Internally to a layer, the work is done by protocol entities which communicate via (peer-to-peer) protocols. 
Reference models for (layered) protocol stacks define what we call the pragmatic aspect of a layered architecture: e.g. layer borders may be used to define visibility rules, the number and order of layers will be fixed and composition rules for protocol data units can be set up. Problems as the one mentioned in the introduction can in many cases be traced back to these pragmatic aspects of a model - which have nothing to do with the concept of layering itself. Our approach closely examines 4 properties common to OSI as well as TCP/IP:

a) services are bound to absolute positions,

b) the interworking between layers can not be influenced,

c) the creation and destruction of instances of protocol entities is done implicitly,

d) if a peer-to-peer protocol is in fact a class of protocols, then negotiation may be required to determine the actually used protocol. The set of options constituting the class is fixed in advance.

a) This point has a limiting as well as a positive aspect: with absolute positioning assumptions can be made on how a given entity can be addressed. On the other side, this excludes arrangements based on a different ordering of the layers. In OSI, for instance, transport services can be found two layer borders downwards from the point of view of an application. This means that the OSI 'association control service element' must translate the (distinguished) name of a remote application to a 'presentation address' which is composed of exactly three items: an address for the transport layer and two selectors one for each of the two intermediate layers.

b) Layer interfaces have a conceptual signification only, and it is left to the engineers how (if ever) these interfaces are realized. There exist however some differences between OSI and TCP/IP: TCP/IP allows an application to use any upper layer-interface directly, while OSI restricts an application to the interface offered by the presentation layer. But in both suites it is not possible to access or manipulate the connections of protocol entities to the layer beneath. Because the ordering and number of layers is fixed there is no need to do so. The mechanism of layer interworking is considered as a local matter.

c) The strategy applied when new instances of protocol entities are to be created and the assignment of the responsibility for this activity is implementation-specific. With Berkeley-Unix there is a special program (the inetd dae- mon [3]) that starts the TCP/IP-applications to which a connection is requested, but other implementations could be considered where only additional buffers etc. are allocated. As a consequence, it is not possible to manipulate instances of protocol entities directly - the creation and destruction of protocol entities is viewed as a side effect of the usage of services.

d) The set of options that can be (down-)negotiated is fixed first by the definition of the protocol and second by the specific implementations involved. No possibility exist to enhance a limited implementation, even if one of the implementations would know how to do the job on the remote system (e.g. because they share the same machine type, the same operating system etc.).

Relaxing one or more of the listed restrictions imposed on a protocol stack will lead to more flexibility, but additional effort is required from the users of a layer. In the next section we will develop and discuss a model that is in contradiction with e.g. the OSI reference model in the four points mentioned above.

\section{A protocol stack environment}

\section{Relaxing layering constraints}

What we propose is a protocol stack environment. Just as TCP/IP and OSI 'model' an architecture, it does not imply the structure of an implementation. Fig. 1 presents an illustration of this model.

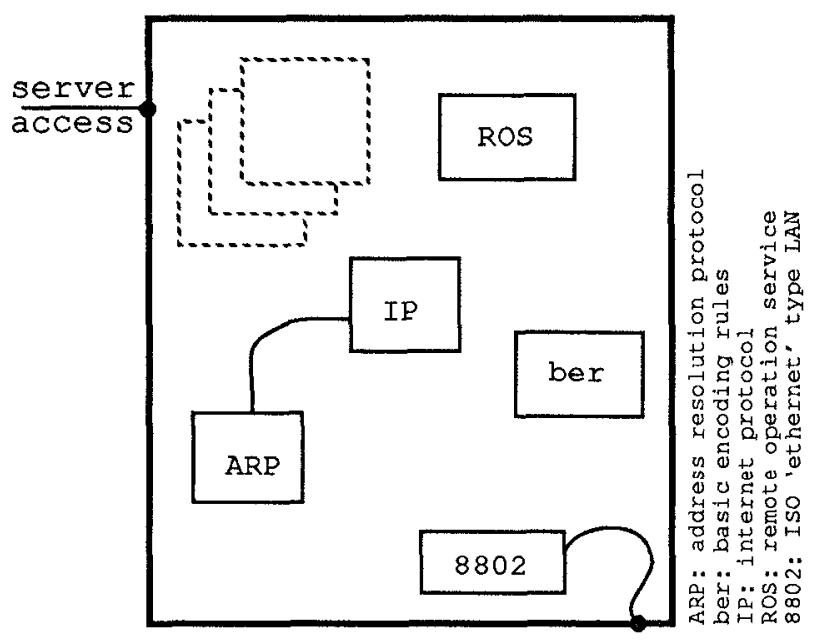

Fig 1. View of the proposed protocol stack environment

The environment can been seen from outside as a communication server: all use of communication services must be made via this server. Inside the server we have a repository 
of static entities (dashed), representing protocol entity types. Instances of these types (solid boxes) may be created on demand. The instances are by nature either 'anchored' or 'free'. Anchored instances (e.g. the box for the ISO 8802 local area network) give immediate access to communication services in the classical sense, e.g. a network service: their availability depends on the hard- and software-configuration of the real system. A free instance, on the other side, must first be connected to an anchored instance before its services can be used. But connections can also be made between 'free' entities (e.g. IP and ARP entity). The advantage here is that these attachments can be unlinked and redefined at any moment, allowing to insert or remove instances at will. Of course the service primitives expected ('lower interface') and the primitives offered ('upper interface') by the two interconnected entities must match.

Up to now we have discussed replies to three of the four points outlined in the preceding section: Services are not bound any more to a predetermined position in a protocol stack (point a) but now must be requested from 'freely floating' protocol instances. Concerning point b), we allow two distinct protocol instances to be connected to each other (provided the primitives match) and this connection to be broken by the environment. And finally (point $c$ ), the protocol environment allows for (and requires) the explicit creation and destruction of protocol instances.

Requirement d) - the extensibility - will be made feasible by the mechanism of downline loading, although some extensions can already be made by letting a local protocol instance manipulate the remote side, e.g. remotely triggering the insertion of an already existing protocol entity. But in some cases this may not be enough. Downline loading will allow to augment the set of entity types available at the remote side. As an example, consider the augmentation of a presentation entity with a new encoding rule (transfer syn$\operatorname{tax}$ ) for abstract data types. The following scenarios will show other possible usages (and limitations) of the proposed protocol environment.

\section{Protocol entities}

In this model classical protocol stacks can be represented as particular protocol entity types. Instantiating e.g. an OSI ROS-entity would create and handle the OSI specific protocol stack underneath. Another 'anchored' protocol entity may offer just the IP services. Instantiating a 'free' TCP entity and connecting it with the IP instance creates a reliable data stream service. Going further, it is possible to instantiate an FTAM application with just the upper OSI layers, then using an 'transport service convergence entity' (RFC 1006) and put this on the TCP entity defined before, which yields an RFC1006 application [5].

Such an application will only interwork with a remote site that knows about RFC1006 applications. Building applications with stacks coming from static protocol suites will always limit their scope to the domain of the sites that understand that architecture. To really make extensive use of the proposed protocol environment we need remote environments fulfilling the same proposed concepts. We do not care how such environments are interconnected but assume that such connections can be made accessible by the means of 'anchored' protocol entities. Important is that all environments understand a generic protocol which is the same as the one used for the access to the environment server. Per default all incoming access to a remote environment will be intercepted by a newly created anchored entity to which a remote 'server' instance is connected (Fig. 2).

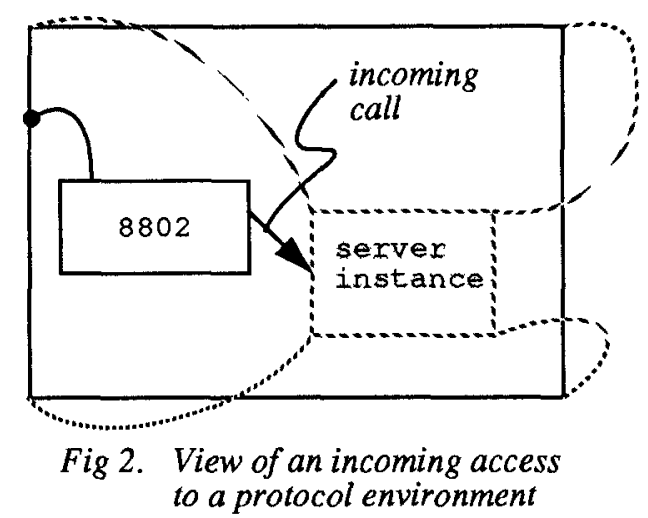

This server entity (dashed box) is virtual: commands issued to it affect the environment it is sitting in, that is the server entity is the environment. An application, together with the required protocol stack, could be remotely constructed out of 'free' protocol entities. Then the built application will take over the connection formerly used between the anchored entity and the server entity.

Using the downline loading capability we can build up nearly arbitrary protocol stacks. Because this can be done in consecutive steps enhancing each other (download, install on both sides, download using new service, install, etc.) this could be called a communication bootstrap.

The price to be paid for this flexibility is an increased complexity when using communication services - an effort that must be made by the application. But by defining entities 
that represent a whole standard protocol stack this can be limited to one additional indirection: the effort needed to locate and to instantiate that protocol entity.

\section{Implementation}

No effort is made to see how this proposed model could be integrated with or made fruitful for OSI or other fixed protocol stacks, although we can try to integrate parts of OSI and TCP/IP technology. The explicit goal of this model is to define 'essential' types of protocol entities which are made visible, whereas other protocol stacks make a principle of hiding a maximum amount of details. Many aspects like addressing, the primitives to be made visible in such a communication server, (in)efficiency considerations etc. have not been discussed yet, and the question remains if such a protocol environment can be realized. Different forms of implementations or attempts in this direction can be envisaged:

a) The generic protocol used to access the server could be of a form similar to PostScript. PostScript [6] allows to control visualisation equipment as well as to use it for printing. The language can be redefined by the means of PostScript itself. A hypothetical 'ComScript' would also be a device- and protocolindependent language used to control a protocol environment but also to use it for communication in general and to redefine and extend the given language.

b) Starting with the question what format should be used for the downline loading we could try to enhance Estelle or LOTOS [7] with the server commands needed. Such a formal description technique would provide a nice generic format for the downline loading of protocol entities.

Both approaches seem to be too ambitious. At the present state of our research not enough is known about the techniques required to handle arbitrary protocol stacks and the techniques needed to implement such an environment. It has been pronounced that Estelle and LOTOS are of too high a level for direct interpretation or use in the automatic generation of executable code. We therefore decided to start with a small 'toy environment' not designed to implement the complete set of suggestions made in this paper. Instead, such an environment allows to experiment with some fundamental problems which arise when protocol stacks are reconfigured dynamically:
- insert into a running protocol stack a new layer (e.g. a multiplexer),

- replace a running layer (e.g. Alternating-Bit by Go-Back-N)

- distribute a running protocol stack on several environments at run time,

- use downline loading for the bootstrap of a protocol stack.

The main difficulty is expected in the synchronisation between two or more participating remote protocol stacks. Not every existing protocol and protocol stack may allow such use.

\section{Related Work}

Topics related to the one discussed here can be found in the border zone of computer communication and distributed operating systems research. Another area where the current structure of protocol stack architectures is re-evaluated is the domain of high-speed networking (e.g. [8]). The main concern, however, are performance figures and related engineering problems, not interworking issues.

The $\mathrm{x}$-Kernel [9] proposes an interesting approach to layer interworking. The kernel is seen as an architecture for implementing network protocols. Protocol entities are represented as a protocol object to which session objects are associated. The $\mathrm{x}$-Kernel does not make any assumptions on the sequence in which protocols will be stacked: a late binding mechanism allows each protocol object to effectively attach itself to another underlying object at run-time. However, the selection of the object to be linked to is pre-determined at compile time. The main advantage is that layer interworking becomes explicit: a small set of primitives uniform for all protocol objects - allows to manipulate the links between these objects. In another paper about the $x$ Kernel [10] a special chapter presents the dynamic removing of layers: in fact a layer is never removed, instead a decision (e.g. based on the packets size) is taken on a per-message basis if a given message can skip the layer or not. The layer itself remains present all the time - particulary in the remote host which cannot know if a messages comes through the so called 'removed' layer or not. Anyhow, the principle interest of the $\mathrm{x}$-Kernel is the demonstration of the advantages of a uniform handling of protocol entities covering the layer interworking.

As another example of architectural support for customized protocol stacks we mention the UNIX System V Transport 
Layer Interface (TLI) [3]. User processes access the transport services via full duplex 'streams': a stream links a user process with a transport provider, TLI being the uniform interface. The transport provider can be represented as a fixed 'stream head' that connects to a device driver. It is now possible to insert (push) between the stream head and the device driver a sequence of modules working on the stream passing them. By the means of multiplexors that handle more than one stream passing through, more complex configurations can be achieved. In this way the kernels services can be augmented e.g. at boot time without the need to recompile the kernel. The control, however, over the placement of intermediate modules is limited in the sense that they form a true stack, allowing manipulations only at the top of the stack.

Both approaches ( $x$-Kernel and TLI) can be used to build and run a variety of different protocol stacks but they remain environments for preconfigured stacks which are difficult to customize at run time by a client application.

\section{The PascalCom Language}

We mentioned above our concern about the feasibility of the proposed protocol stack environment: more research is needed to find stack composition technics. With this goal in mind we designed a toy environment based on a language we called PascalCom and an easily portable run time system. At the time of writing we are in the last phase of testing and debugging the compiler in parallel with the run time system for several platforms (Sun, Transputer and PC).

PascalCom is an extension of the Pascal programming language. Pascal was used as the base language because it's semantic is well understood and surprises must be expected for extensions only. Moreover small Pascal compilers/interpreters susceptible to incorporate the necessary extensions at a reasonable effort are freely available (actually a revised Pascal-S is used [11]). PascalCom explicitly targets the study of protocol stack implementations in the context described so far and may be viewed as one possible way to implement some rudimentary form of flexible protocol stacks. The following Section provides a quick overview of PascalCom.

When specifying the language we applied the principle to keep the number of additional primitives as small as possible. Enhancements were made in three areas:

some minor extensions concerning data types,
- the creation and control of parallel processes,

- communication between parallel processes.

\section{a) PascalCom data types}

Care has been taken not to arbitrarily introduce fancy new data types. Beside a small completion of the standard list of types (e.g. byte and time) there is only one really new type: the variable array varray. This extension is needed to provide a formal means to support dynamic allocation without losing strong typing, which is of particular interest for the implementation of protocol entities. Strings are internally handled as variable arrays of characters:

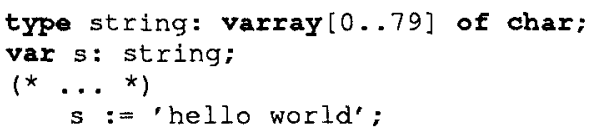

A variable of type varray can be regarded as a parametric record having three fields being defined as follows:

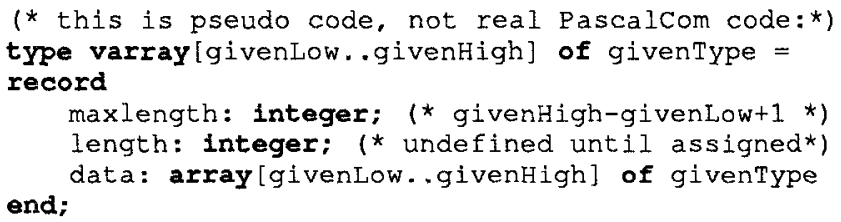

Mainly when protocol-data-units must be represented, such a type is often involved implicitly:

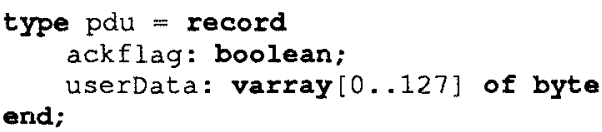

The varray type also has a special pointer form, allowing to allocate dynamically an amount of memory not specified at program time:

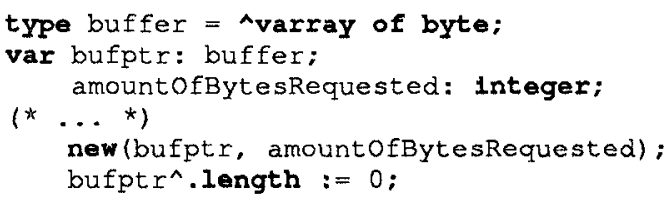

\section{b) PascalCom processes/agents}

Generally the code of a program does not only define a flow of control, but contains also some state information (global variables etc.). We call this extended view of a program (that is the compiled code together with the state of execution) an agent. Formally, this correspond to replace the keyword program by the keyword agent.

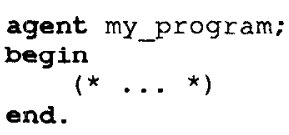

The notion of an 'agent' will serve to formally represent dif- 
ferent process types: an instance of an agent is called a process. PascalCom has built-in primitives to start and stop a process and others that allow to turn an agent (which is an ordinary data structure) into a process and vice-versa.

The definition of a local agent must precede the variable declarations, because child processes may not access the parent's data (the exchange of information therefore is possible only via messages). When a new process is to be created, it is the task of the parent to furnish the needed 'workspace' (similar to Modula-2 for coroutines [12]).

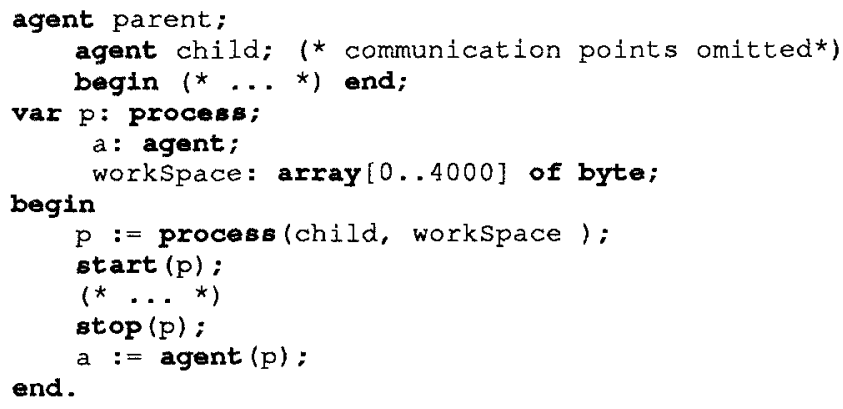

The repeated creation of child processes will eventually lead to a tree of processes. When such a tree of processes is transformed into an agent this will be carried out recursively on all subprocesses but will yield only one agent. On instantiation of this agent the whole process tree is recreated. All processes run in parallel: no special priority is given to a parent process.

\section{c) PascalCom communication primitives}

Communication in PascalCom follows to a large degree the idea proposed in MEX (Modula-2 EXtented [13]): communication between processes is synchronous and may be used to exchange data between gates. The primitives for synchronisation are the accept and the of fer construct which will block if the other side is not ready. Both primitives may be used in a select statement, thus attempting a synchronisation on one of the entries ("symmetric select"). Some important changes with respect to MEX have been made:

- gates are typed (in direction as well as for the data type)

- the offer performs a data copy (in MEX only pointers are exchanged)

- gates have a very narrow scope (gates are global in MEX).

The gates used in PascalCom are inspired more by the concept of interaction points found in Estelle than the gate concept found in MEX: the PascalCom gates in fact should be regarded as 'half-gates' because they must be connected together before a synchronisation becomes possible. A connection between gates can be broken at any moment by a di sconnect. An advantage of the strong typing of gates is the differentiation between input gates (source) and output gates (sink). This allows an additional syntactic check when gates are used in the accept, offer and connect primitive.
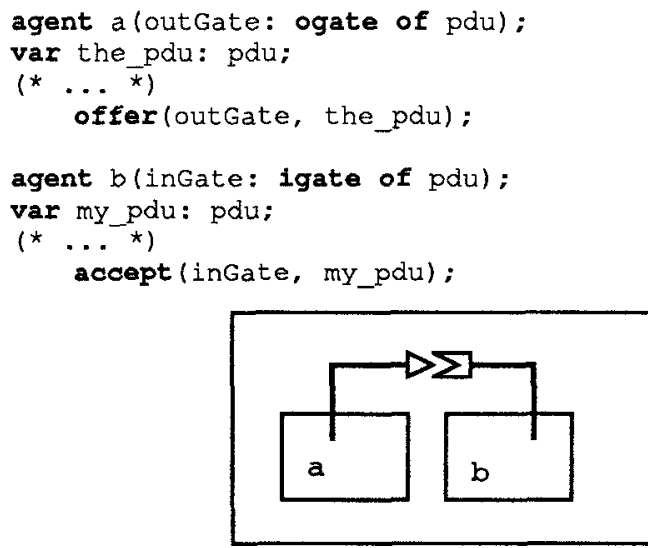

Fig 3. Possible configuration of the two processes

If two processes have corresponding synchronisation requests and the two (half-)gates used are connected and this synchronisation is 'chosen' by the environment, then the data in the offer statement is copied to the accepting side and both processes may continue their execution. Of course the data parameter for accept must be a variable that is compatible with the gate's data type.

The type associated to a gate may not contain pointer types, gates, processes and agents. Gates can nevertheless be used to transfer the content of an agent variable under the condition that the program provides an adequate external representation.

The symmetric select allows the mix of of fers, accepts and timed entries. Each entry may be guarded by a boolean expression. The parameter of the offer primitive is evaluated each time a synchronisation is attempted, not just when the offered entry is selected (MEX has a different semantic here).

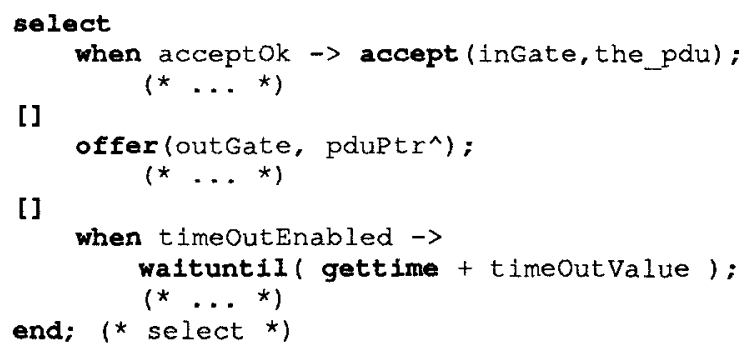


One of the select-entries ready for synchronisation will be chosen by the environment and the code following this entry is executed.

Gates are only valid in the direct relationship of parent/child processes: the parent is the owner of a gate - the gate may only be used by direct off-springs. The only moment to pass gates to a child is at the time a process is started (gates are the only parameters allowed in the parameter list of an agent definition): the actual (gate-)parameter must be a variable owned by the parent (and not a gate inherited in turn):

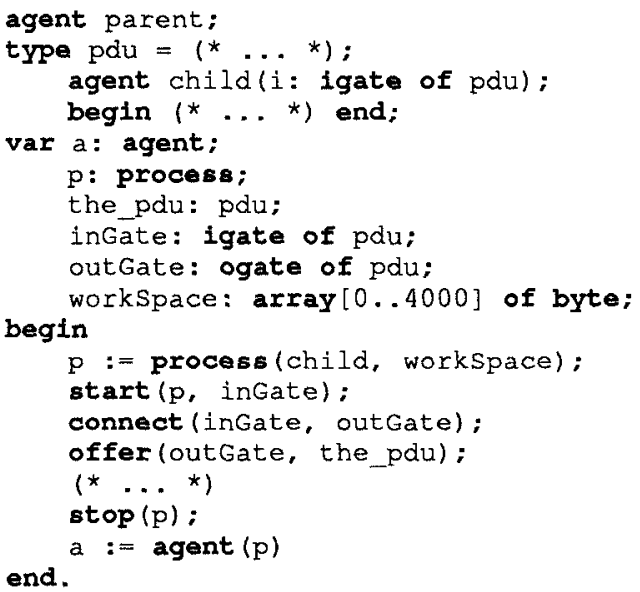

If several children share the same gate then one child will be selected randomly in the case of multiple possible synchronisations.

\section{d) Discussion of PascalCom}

PascalCom processes can be used to implement a simple form of the protocol entities proposed in this paper. The important aspect is the way processes can be handled: PascalCom allows to tightly control their execution. Processes can be 'frozen' into agents such that they may be either locally duplicated or moved to some other environment which enables downline loading. Also important is the aspect of controlling the interconnections between child processes. Gates can be linked together or be separated: so it will be possible for the parent to insert other processes in an existing connection.

Our PascalCom compiler generates code for a very simple virtual machine. The communication and the process handling primitives are accessible through 'system calls' of a primitive operating system (called ComOs) and are thus separated from the interpreter. One architectural goal of this work item is the possibility to implement the ComOs using the primitives offered by PascalCom itself. This is an elegant approach having recursive environments as it allows to download enhanced protocol environments, making the communication bootstrap more general. We feel that the primitives and concepts introduced in PascalCom are sufficient powerful for allowing this.

As an example we include in the appendix the code for an 'alternating bit protocol' entity: it implements a full duplex reliable data stream. Additionally if offers a control access that allows for either a reset of the protocol entity or a flush with end-to-end significance.

\section{Conclusion}

In this paper we investigated the pragmatics attached to the (static) layer concept in OSI and TCP/IP. Based on this observation we outline a model for a flexible protocol architecture: Communication services are offered by a protocol environment accessible through a generic protocol. Inside this environment we have protocol entities that can (and must) be explicitly created and destroyed, the interconnection of these entities can be dissolved and redefined at will. This allows a generic communication bootstrap procedure, potentially solving many interworking problems between static protocol suites.

A consequence of this approach is the blurring of the strict layer model. In fact, the layer model is now viewed as just one way to organise communication tasks. This relaxed view already found its way into OSI where in layer 7 we have different floating 'service elements', showing an impressive number of interconnection patterns. The effort in Open Distributed Processing (ODP) goes in the same direction when the process migration is envisaged.

Before one can hope to have such a general protocol environment (e.g. controllable by something like ComScript), more understanding about the management of running protocol stacks is needed. As a first step, we will use PascalCom as a testbed for the 4 basic experiments listed in this paper.

\section{Acknowledgement}

I gratefully acknowledge Dave Robson for providing us the sources of their modified Pascal-S compiler. Thanks go also to Eduardo Solana and Muhugusa Murhimanya who implement the compiler extensions and the execution environment, and especially to Jürgen Harms, supporting me in the writing of this paper. I am also grateful for the remarks of 
the reviewers - not only because of the structural improvements proposed, but also for the objective comments very welcome in a research project at an early stage.

\section{Bibliography}

[1] CCITT: Data Communication Networks - Open System Interconnection (OSI) Model and Notation, Service definition; Blue Book recommendations X.200-X.219; International Telecommunication Union 1989.

[2] W. Stallings: Handbook of Computer-Communications Standards - Volume 3: The TCPIIP Protocol Suite; Stalling/Macmillan 1989.

[3] W. Richard Stevens: Unix Network Programming; Prentice-Hall 1990.

[4] R. van Renesse, A.S. Tanenbaum: The Evolution of a Distributed Operating System (Amoeba); in "Progress in Distributed Operating Systems and Distributed Systems Management"; Lecture Notes in Computer Science 433, pp. 1-12; Springer 1990.

[5] Marshall T. Rose: The Open Book - A practical perspective on OSI; Prentice-Hall 1989.

[6] Adobe: PostScript Language - Reference Manual; Addison-Wesley 1990.

[7] Dieter Hogrefe: Estelle, LOTOS und SDL; Springer 1989.

[8] David D. Clark, David L. Tennenhouse: Architectural Considerations for a New Generation of Protocols; in "Communications Architectures \& Protocols"; SIGCOMM '90 Symposium, pp. 200-208; ACM 1990.

[9] Norman C. Hutchinson, Larry L. Peterson: The $x$-Kernel: An Architecture for Implementing Network Protocols; in IEEE Transactions on Software Engineering, Vol. 17, No. 1, pp. 64-76; IEEE 1991.

[10] Norman C. Hutchinson, Larry L. Peterson, Mark B. Abott, Sean O'Malley: RPC in the X-Kernel: Evaluating New Design Techniques; in "Proc. Twelfth ACM Symposium on Operating System Principles”, pp. 91101; ACM 1989.

[11] Michael Rees, Dave Robson: Practical Compiling with Pascal-S; Addison-Wesley 1988.

[12] Niklaus Wirth: Programming in Modula-2; Springer 1983.

[13] Giovanni Conti: Méthodologie d' implantation des protocoles de communication - Ph.D. thesis No 842; Ecole Polytechnique Fédérale de Lausanne, 1990. 

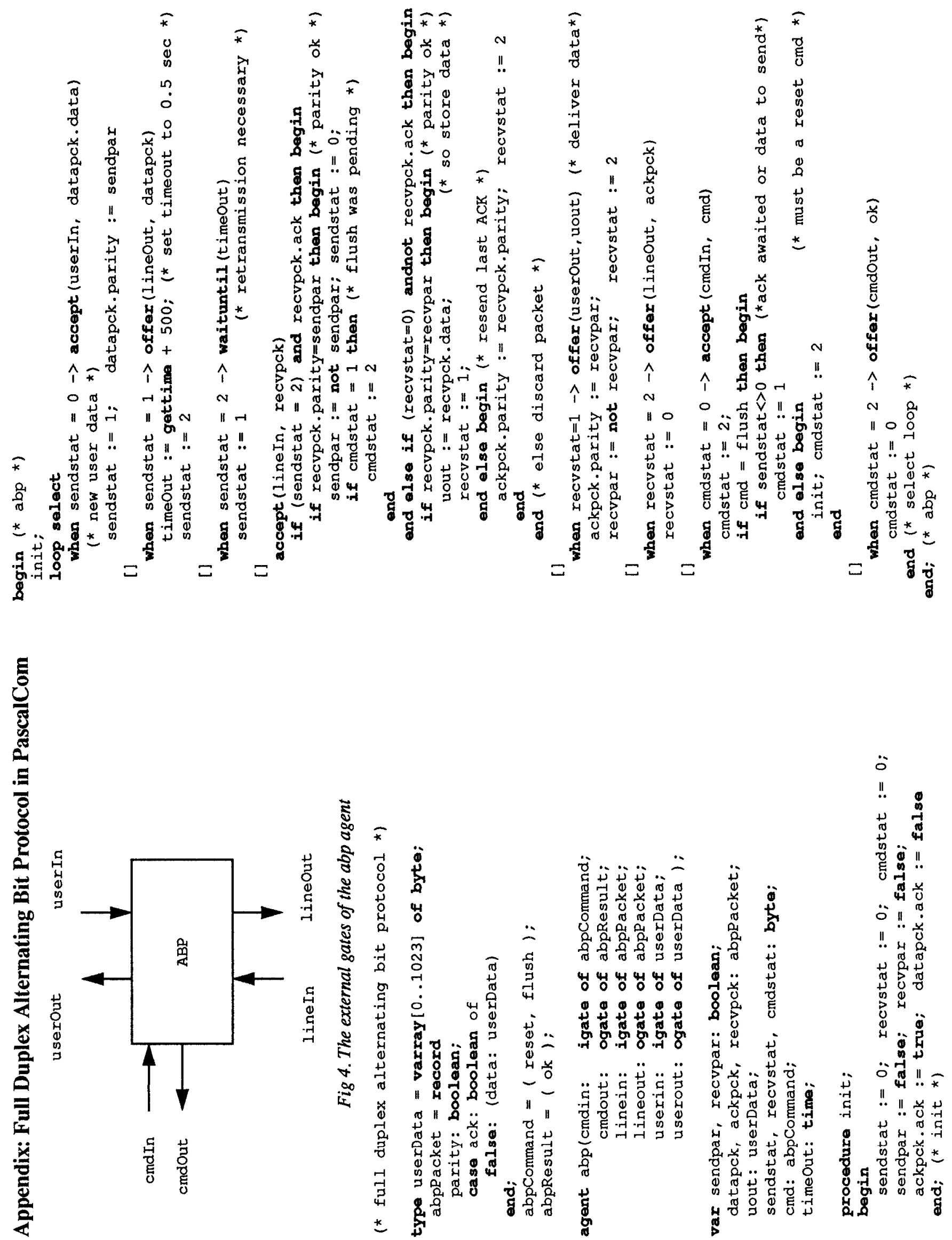